\section{Commentary: Mitral bioprosthesis degeneration: Looking for a benchmark to tailor the correct procedure to the patient}

\author{
Francesco Formica, MD, and \\ Francesco Nicolini, MD, PhD
}

In recent years, following the increasingly promising clinical results of transcatheter aortic valve implantation, alternative techniques to conventional surgery are emerging to replace a deteriorated mitral bioprosthesis by means of transcatheter mitral valve implantation (VIV). ${ }^{1}$ However, mitral VIV procedures are not without procedural risks, ${ }^{2,3}$ and therefore it is mandatory to select those high-risk patients who could benefit from VIV techniques.

Javadikasgari and colleagues ${ }^{4}$ from the Cleveland Clinic reported an interesting and timely analysis of patients undergoing reoperation for mitral bioprosthesis structural valve deterioration with the aim of assessing early- and long-term outcomes and risk factors for mortality for replacement of the isolated and concomitant mitral bioprosthesis. The great value of this study is the attempt to establish a benchmark for this difficult population. The authors analyzed data on the replacement of 525 bioprosthetic mitral valves over a period between 1990 and 2017. Among these patients, 133 received an isolated bioprosthetic valve replacement (BVR) and 392 underwent a combined BVR with other cardiac procedures. Mortality was significantly lower in the isolated replacement group than in the nonisolated replacement group $(0.75 \%$ vs $7.1 \%$ ), and this trend was also observed in the matched population. Long-term survival was significantly better in isolated BVR than in combined BVR. The key message was that isolated BVR is safe, whereas the combined operation is still affected by the negative outcome and

\footnotetext{
From the Cardiac Surgery Unit, Department of Medicine and Surgery, University Hospital of Parma, University of Parma, Parma, Italy.

Disclosures: The authors reported no conflicts of interest.

The Journal policy requires editors and reviewers to disclose conflicts of interest and to decline handling or reviewing manuscripts for which they may have a conflict of interest. The editors and reviewers of this article have no conflicts of interest.

Received for publication Sept 29, 2020; revisions received Sept 29, 2020; accepted for publication Sept 30, 2020; available ahead of print Oct 9, 2020.

Address for reprints: Francesco Formica, MD, UO Cardiochirurgia, Azienda Ospedaliera Universitaria di Parma, Via Antonio Gramsci, 14, 43126, Parma, Italy (E-mail: francesco_formica@fastwebnet.it).

J Thorac Cardiovasc Surg 2022;163:1816-7

$0022-5223 / \$ 36.00$

Copyright (C) 2020 by The American Association for Thoracic Surgery

https://doi.org/10.1016/j.jtcvs.2020.09.125
}

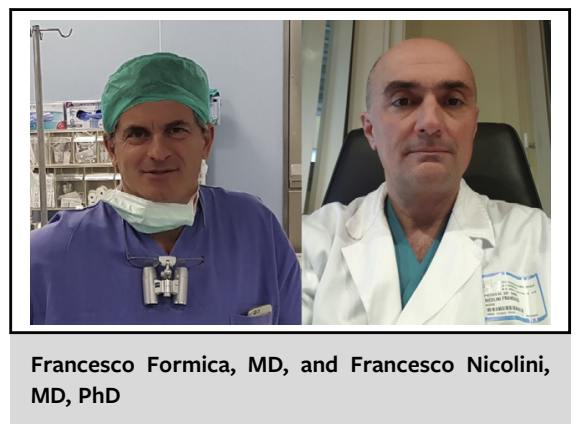

CENTRAL MESSAGE

A full evaluation of patients with mitral bioprosthetic structural valve deterioration is of great importance to better select which patients would benefit from an either a surgical or VIV procedure.

therefore less-invasive alternative procedures should be ensured.

The authors identified 392 patients who underwent concomitant mitral BVR over 27 years, or approximately 14 patient-years from a large-volume center. These patients should be inferior in medium-volume centers. This suggests that in clinical practice this population represents a low number. Furthermore, the observed/expected mortality ratio for isolated mitral BVR was $0.12(0.75 \%$ vs the Society of Thoracic Surgeons expected mortality score of $6.1 \%$ ), whereas the observed/expected for combined mitral BVR was $11 \%$ versus predicted Society of Thoracic Surgeons mortality score of $9.4 \%$, although the latter data were obtained from 55 of 392 patients. The complexity of the primary cardiac operation and the clinical condition at the time of reoperation should guide the establishment of a surgical benchmark. For example, patients with previous mitral valve replacement and triple coronary artery bypass grafting can undergo isolated mitral BVR through right minithoracotomy access with encouraging results. ${ }^{5}$ In contrast, patients who have received an isolated mitral valve replacement can be dramatically frail at the time of an isolated BVR. For this subset of patients, isolated mitral BVR should be considered a high-risk procedure. The problem is not solved yet. In fact, the study of Javadikasgari and colleagues does not focus on the type of primary surgery, preoperative conditions, and mode of entry to the chest, but focuses primarily on the type of reoperation, both isolated and combined mitral BVR. Naturally, the results reported 
by the authors are of fundamental importance and represent an indispensable element to better select which patients would benefit from a surgical procedure or VIV; however, this patient population should be fully analyzed by a multidisciplinary team. ${ }^{6}$ Otherwise, some fundamental information for the definition of the benchmark could be underestimated or not taken into consideration.

\section{References}

1. Whisenant B, Kapadia SR, Eleid MF, Kodali SK, McCabe JM, Krishnaswamy A, et al. One-year outcomes of mitral valve-in-valve using the SAPIEN 3 transcatheter heart valve. JAMA Cardiol. July 29, 2020 [Epub ahead of print].

2. Yoon SH, Bleiziffer S, Latib A, Eschenbach L, Ancona M, Vincent F, et al. Predictors of left ventricular outflow tract obstruction after transcatheter mitral valve replacement. JACC Cardiovasc Interv. 2019;12:182-93.
3. Yoon SH, Whisenant BK, Bleiziffer S, Delgado V, Dhoble A, Schofer N, et al. Outcomes of transcatheter mitral valve replacement for degenerated bioprostheses, failed annuloplasty rings, and mitral annular calcification. Eur Heart J. 2019; 40:441-51.

4. Javadikasgari H, Chemtob RA, Gillinov MA, Pettersson GB, Lowry AM Desai MY, et al. Outcomes of mitral valve re-replacement for bioprosthetic structural valve deterioration. J Thorac Cardiovasc Surg. 2022;163:1804-12.e5.

5. Daemen JHT, Heuts S, Olsthoorn JR, Maessen JG, Sardari Nia P. Right minithoracotomy versus median sternotomy for reoperative mitral valve surgery: a systematic review and meta-analysis of observational studies. Eur J Cardiothorac Surg. 2018;54:817-25.

6. Bonow RO, O'Gara PT, Adams DH, Badhwar V, Bavaria JE, Elmariah S, et al. 2019 AATS/ACC/SCAI/STS expert consensus systems of care document: operator and institutional recommendations and requirements for transcatheter mitral valve intervention: a joint report of the American Association for Thoracic Surgery, the American College of Cardiology, the Society for Cardiovascular Angiography and Interventions, and the Society of Thoracic Surgeons endorsed by the Heart Failure Society of America. J Thorac Cardiovasc Surg. 2020;160:72-92. 